\title{
Heterogeneous structures studied by an interphase elasto-plastic damaging model
}

\author{
G. Giambanco, G. Fileccia Scimemi \& A. Spada \\ Department of Civil, Environmental, Aerospace and Materials' Engineering \\ University of Palermo - Palermo, Italy
}

\begin{abstract}
Heterogeneous materials present a mechanical response strongly dependent on the static and kinematic phenomena occurring in the constituents and at their joints. At the mesoscopic level the interaction between the units is simulated by mean of apposite mechanical devices such as the zero thickness interface model where contact tractions and displacement discontinuities are the primary static and kinematic variables respectively. In heterogeneous materials the response also depends on joint internal stresses. The introduction of internal stresses brings to the interphase model or an enhancement of the classical zero-thickness interface. With the term 'interphase' we shall mean a layer separated by two physical interfaces from the bulk material or a multilayer structure with varying properties and several interfaces. Different failure conditions can be introduced for the physical interfaces and for the joint material. The interphase model has been implemented in an open-source research-oriented finite element analysis program for 2D applications.
\end{abstract}

\section{INTRODUCTION}

The mechanical response of all those structures that are constituted by heterogeneous materials is dependent by different static and kinematic phenomena occurring in each constituent and at their joints. Material degradation due to nucleation, growth and coalescence of microvoids and microcracks is usually accompanied by plastic deformations as decohesion and sliding that cause strain softening and induced anisotropy.

The mesoscopic approach is by now the most diffused technique to understand this kind of materials, because it overcomes the problems associated with the strong simplifications that have to be introduced when the macroscopic approach is applied. In particular, with the mesoscopic approach all the material constituents are modelled individually and their interactions are regulated by using appropriate devices able to reproduce the inelastic phenomena that usually occur at the physical interfaces. In the last decades zero-thickness interface elements (ZTI) have been applied in several engineering applications due to their simple formulation and to their easiness to be implemented in finite element codes.

The interface constitutive laws are expressed in terms of contact tractions and displacement discontinuities which are considered as generalized joints strains. In order to model the nonlinear behaviour caused by plastic deformations and damage evolution the constitutive laws of the interface elements are formulated making use of concepts borrowed by theory of Plasticity and Continuum Damage Mechanics.

In many cases the structural response depends also on internal stresses and strains within the joint. It is sufficient to think to the fracture that appears in the middle of masonry blocks caused by the horizontal tangential contact stresses between the mortar and the block when a masonry assembly is subjected to a pure compressive load. These tangential stresses cannot be captured by the classical ZTI model. Therefore, the usual assumption used in zerothickness interface elements, where the response is governed by contact stress components, may require a correction by introducing the effect of the internal stresses into the analysis. This enhancement of the ZTI is known as interphase model, for the first time proposed by Giambanco and Mròz (2001).

The interphase element has been formulated by authors as a new contact element and introduced in a scientific oriented finite element code. Patch tests have been carried out in elasticity to investigate the numerical performance and convergence of the element. All the results are shown in the paper written by Giambanco el al. (2012). In particular, in that paper is shown how strategies such as the Reduced Selective Integration or the Enhanced Assumed Strain methods are necessary to avoid shear locking effects of the element.

In this work the interphase element is implemented for nonlinear applications by introducing 
separate limit conditions for the joint bulk material and for the physical interfaces. The overall model is thermodynamically consistent and the flow rules are derived by applying the Lagrangian method. With the aim to show the effectiveness of the model the interphase constitutive laws have been implemented in an open-source research-oriented finite element analysis program for $2 \mathrm{D}$ applications and by using the Selective Reduced Integration.

\section{GENERAL ASSUMPTIONS AND THERMODYNAMICS.}

Let us consider, in the Euclidean space $\mathfrak{R}^{3}$ referred to the orthonormal frame $\left(O, \mathbf{i}_{1}, \mathbf{i}_{2}, \mathbf{i}_{3}\right)$, a structure formed by two adherents $\Omega^{+}, \Omega^{-}$connected by a third material $\Omega$ in contact with the two bodies by means of the two physical interfaces $\Sigma^{+}$and $\Sigma^{-}$respectively, as in Figure 1.
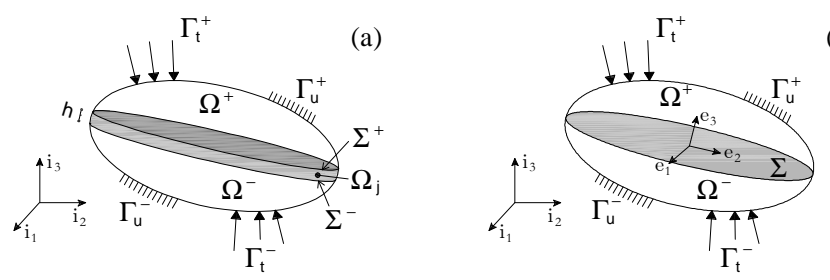

Figure 1. (a) Mechanical scheme of a third body interposed between two adherents; (b) Interphase mechanical scheme.

It is assumed that the thickness $h$ of the joint is small if compared with the characteristic dimensions of the bonded assembly.

The boundary of the two adherents is divided in the two parts $\Gamma_{u}^{ \pm}$and $\Gamma_{t}^{ \pm}$, where kinematic and loading conditions are specified respectively.

The joint interacts with the two adherents through the following traction components:

$$
\mathbf{t}^{ \pm}=t_{1}^{ \pm} \mathbf{e}_{1}+t_{2}^{ \pm} \mathbf{e}_{2}+t_{3}^{ \pm} \mathbf{e}_{3}
$$

which can be considered as the external surface loads for the joint.

In Equation $1 \mathbf{e}_{1}, \mathbf{e}_{2}$ and $\mathbf{e}_{3}$ are the unit vectors of the local reference system, with $\mathbf{e}_{3}$ oriented along the normal to the middle surface $\Sigma$ and directed towards the adherent $\Omega^{+}$. The joint can be regarded as an interphase model. It is assumed that the fibers inside the interphase and directed along $\mathbf{e}_{3}$ are maintained rectilinear during the deformation process. In view of this hypothesis the interphase displacement field $\mathbf{u}$ can be easily obtained from the displacement $\mathbf{u}^{+}$and $\mathbf{u}^{-}$of the interfaces $\Sigma^{+}$and $\Sigma^{-}$, thus

$\mathbf{u}\left(x_{1}, x_{2}, x_{3}\right)=\left(\frac{1}{2}+\frac{x_{3}}{h}\right) \mathbf{u}^{+}\left(x_{1}, x_{2}\right)+\left(\frac{1}{2}-\frac{x_{3}}{h}\right) \mathbf{u}^{-}\left(x_{1}, x_{2}\right)$ with $x_{1}, x_{2}$ and $x_{3}$ the Cartesian coordinates in the orthonormal frame $\left(O, \mathbf{e}_{1}, \mathbf{e}_{2}, \mathbf{e}_{3}\right)$.

Since the thickness of the joint is generally small if compared to the characteristic dimensions of the adherents, we can assume the strain state $\boldsymbol{\varepsilon}$ uniform along the $\mathbf{e}_{3}$ direction and given by:

$\boldsymbol{\varepsilon}\left(x_{1}, x_{2}\right)=\frac{1}{h} \int_{-h / 2}^{h / 2} \nabla^{s} \mathbf{u}\left(x_{1}, x_{2}, x_{3}\right) d x_{3}$

Substituting the Equation 2 we have:

$\boldsymbol{\varepsilon}\left(x_{1}, x_{2}\right)=\frac{1}{2 h}([\mathbf{u}] \otimes \mathbf{n}+\mathbf{n} \otimes[\mathbf{u}])+\frac{1}{2} \nabla^{s}\left(\mathbf{u}^{+}+\mathbf{u}^{-}\right)$

where $[\mathbf{u}]=\mathbf{u}^{+}-\mathbf{u}^{-}, \mathbf{n}$ is the unit normal vector to the interphase plane and $\nabla^{s}$ is the symmetric gradient operator defined as $\nabla^{s}=\frac{1}{2}\left(\nabla+\nabla^{T}\right)$.

Let us note that in the interphase model the joint curvatures generated by displacement field (2) and the related flexural effect are neglected.

Equilibrium equations are derived by applying the principle of virtual displacements (PVD) that asserts that the external work produced by the contact tractions equals the internal work developed in the joint. According to the hypothesis of a constant strain state, by applying the divergence theorem and assuming that $\Sigma=\Sigma^{+}=\Sigma^{-}$, the PVD leads to the following local equilibrium relations of the interphase model:

$$
\begin{array}{ll}
\mathbf{t}^{+}-\boldsymbol{\sigma} \cdot \mathbf{n}+\frac{h}{2} \operatorname{div} \boldsymbol{\sigma}=\mathbf{0} ; & \\
\mathbf{t}^{-}+\boldsymbol{\sigma} \cdot \mathbf{n}+\frac{h}{2} \operatorname{div} \boldsymbol{\sigma}=\mathbf{0} & \\
\mathbf{m} \cdot \boldsymbol{\sigma}=\mathbf{0} & \text { on } \Sigma,
\end{array}
$$

The basic kinematical hypotheses are the additive decomposition of total strain in the internal $(i)$ and contact $(c)$ parts and, for the contact strain only, a further decomposition in elastic $(e)$ and inelastic $(p)$ parts:

$\boldsymbol{\varepsilon}=\boldsymbol{\varepsilon}^{i}+\boldsymbol{\varepsilon}^{c}$

$\boldsymbol{\varepsilon}^{c}=\boldsymbol{\varepsilon}^{c e}+\boldsymbol{\varepsilon}^{c p}$

with

$\boldsymbol{\varepsilon}^{i}=\mathbf{A} \boldsymbol{\varepsilon} \mathbf{A}$

being $\mathbf{A}=\mathbf{I}-\mathbf{n} \otimes \mathbf{n}$ the unit second order tensor.

In order to comply with thermodynamic requirements, the interphase Helmholtz free energy is introduced in the following form:

$$
\begin{array}{r}
\Psi\left(\boldsymbol{\varepsilon}^{i}, \boldsymbol{\varepsilon}^{c}, \boldsymbol{\varepsilon}^{c p}, \boldsymbol{\omega}, \boldsymbol{\xi}_{d}, \boldsymbol{\xi}_{p}\right)=\Psi^{i}\left(\boldsymbol{\varepsilon}^{i}, \boldsymbol{\omega}, \boldsymbol{\xi}_{d}\right)+ \\
+\Psi^{c}\left(\boldsymbol{\varepsilon}^{c}, \boldsymbol{\varepsilon}^{c p}, \boldsymbol{\xi}_{p}\right)+\Psi^{i, c}\left(\boldsymbol{\varepsilon}^{i}, \boldsymbol{\varepsilon}^{c}, \boldsymbol{\varepsilon}^{c p}, \boldsymbol{\omega}\right)
\end{array} ;
$$


where $\Psi^{i}$ and $\Psi^{c}$ represent the free energies related to the internal and contact parts of the strain state respectively and $\Psi^{i c}$ is the mixed term of the free energy which takes into account the co-presence of the contact and internal strains. $\xi_{d}$ and $\xi_{p}$ are the damage and plastic internal variables, respectively.

The principle considered for developing the constitutive laws is that damage occurs in the bulk material, therefore the damage tensor $\boldsymbol{\omega}$ appears in the two terms of the total free energy that are functions of the internal strains also. In this way the constitutive model takes into account the onset of microvoids and fractures along the thickness of the joint. On the other hand, debonding of the joint from the adherents, sliding and fractures developing on surfaces parallel to the middle plane of the interphase are modelled using elastoplasticity and the inelastic contact strains $\varepsilon^{c p}$ are the related internal variables.

In this work a single scalar damage variable $\omega$ governs the loss of stiffness of the bulk material. It ranges from 0 to 1 , with the inferior and superior limits having the meaning of a pristine and a fully damaged bulk material, respectively.

The explicit expression of the components of the free energy is given below:

$$
\begin{aligned}
& \begin{array}{l}
\Psi^{i}=\frac{1}{2}(1-\omega)\left[\lambda \operatorname{tr}^{2}\left(\boldsymbol{\varepsilon}^{i}\right)+2 \mu \boldsymbol{\varepsilon}^{i}: \boldsymbol{\varepsilon}^{i}\right]+ \\
-h_{d}\left[\xi_{d}+\ln \left(1-\xi_{d}\right)\right]
\end{array} \\
& \Psi^{c}=\frac{1}{2}\left[\lambda \operatorname{tr}^{2} \boldsymbol{\varepsilon}^{c e}+2 \mu \boldsymbol{\varepsilon}^{c e}: \boldsymbol{\varepsilon}^{c e}\right]+\frac{1}{2} h_{p} \xi_{p}^{2} \\
& \Psi^{i, c}=(1-\omega) \lambda \operatorname{tr}\left(\boldsymbol{\varepsilon}^{\mathrm{i}}\right) \operatorname{tr} \boldsymbol{\varepsilon}^{\mathrm{ce}}
\end{aligned}
$$

where $\lambda$ and $\mu$ are the Lamè's constants, $h_{d}$ is a material parameter which governs the softening response associated to the damage onset and growth, and $h_{p}$ is a material parameter specifying isotropic hardening/softening interface response.

\section{STATE EQUATIONS AND FLOW RULES.}

In order to derive the interphase constitutive equations, the second principle of thermodynamics, taking into account also the balance equation (first principle) can be applied in the form of the ClausiusDuhem inequality. This inequality for isothermal purely mechanical evolutive process reads as

$$
D=\boldsymbol{\sigma}: \dot{\boldsymbol{\varepsilon}}-\dot{\Psi} \geq 0
$$

where $D$ is the interphase dissipation (for unit surface) or net entropy production.

From the assumed form of the Helmholtz free energy (Eq. 10) its general rate has the following expression:

$$
\begin{aligned}
& \dot{\Psi}=\left(\frac{\partial \Psi^{i}}{\partial \boldsymbol{\varepsilon}^{i}}+\frac{\partial \Psi^{i, c}}{\partial \boldsymbol{\varepsilon}^{i}}\right): \dot{\boldsymbol{\varepsilon}}^{i}+\left(\frac{\partial \Psi^{c}}{\partial \boldsymbol{\varepsilon}^{c}}+\frac{\partial \Psi^{i, c}}{\partial \boldsymbol{\varepsilon}^{c}}\right): \dot{\boldsymbol{\varepsilon}}^{c}+ \\
& +\left(\frac{\partial \Psi^{c}}{\partial \boldsymbol{\varepsilon}^{c p}}+\frac{\partial \Psi^{i, c}}{\partial \boldsymbol{\varepsilon}^{c p}}\right): \dot{\boldsymbol{\varepsilon}}^{c p}+\left(\frac{\partial \Psi^{i}}{\partial \omega}+\frac{\partial \Psi^{i, c}}{\partial \omega}\right): \dot{\omega}+ \\
& +\frac{\partial \Psi^{i}}{\partial \xi_{d}} \dot{\xi}_{d}+\frac{\partial \Psi^{c}}{\partial \xi_{p}} \dot{\xi}_{p}
\end{aligned}
$$

Particularizing Equation 14 for a purely elastic incremental deformation process $\left(\dot{\boldsymbol{\varepsilon}}^{c p}=\mathbf{0}\right.$, $\dot{\omega}=\dot{\xi}_{d}=\dot{\xi}_{p}=0$ ), assuming the decomposition of the stress state similar to that used for the strain state

$\boldsymbol{\sigma}=\boldsymbol{\sigma}^{i}+\boldsymbol{\sigma}^{c}, \quad$ being $\boldsymbol{\sigma}^{i}=\mathbf{A} \boldsymbol{\sigma} \mathbf{A}$

and considering the adopted expressions of the free energy parts (Eqs. 11-13), the elastic stress-strain equations can be derived, thus

$$
\begin{aligned}
& \sigma^{i}=(1-\omega)\left\{\lambda\left[\operatorname{tr}\left(\boldsymbol{\varepsilon}^{i}\right)+\operatorname{tr}\left(\boldsymbol{\varepsilon}^{c}-\boldsymbol{\varepsilon}^{c p}\right)\right] \mathbf{A}+2 \mu \boldsymbol{\varepsilon}^{i}\right\} \\
& \sigma^{c}= \lambda\left[(1-\omega) \operatorname{tr}\left(\boldsymbol{\varepsilon}^{i}\right)+\operatorname{tr}\left(\boldsymbol{\varepsilon}^{c}-\boldsymbol{\varepsilon}^{c p}\right)\right](\mathbf{I}-\mathbf{A})+ \\
&+2 \mu\left(\boldsymbol{\varepsilon}^{c}-\boldsymbol{\varepsilon}^{c p}\right) \\
& \chi_{p}= h_{p} \xi_{p} \\
& \chi_{d}=h_{d} \frac{\xi_{d}}{1-\xi_{d}} \\
& \zeta=\frac{1}{2}\left[\lambda \operatorname{tr}^{2}\left(\boldsymbol{\varepsilon}^{i}\right)+2 \mu \boldsymbol{\varepsilon}^{i}: \boldsymbol{\varepsilon}^{i}\right]+\lambda \operatorname{tr}\left(\boldsymbol{\varepsilon}^{i}\right) \operatorname{tr}\left(\boldsymbol{\varepsilon}^{c}-\boldsymbol{\varepsilon}^{c p}\right)
\end{aligned}
$$

where $\chi_{p}$ and $\chi_{d}$ are the static variables conjugate of the internal variables $\xi_{p}$ and $\xi_{d}$ respectively, and $\zeta$ the thermodynamic force conjugate of the damage variable $\omega$.

Making use of the elastic strain-stress equation and of the previous positions, the final expression of the instantaneous dissipation is obtained:

$$
D=\boldsymbol{\sigma}^{c}: \dot{\boldsymbol{\varepsilon}}^{c p}-\chi_{p} \dot{\xi}_{p}-\chi_{d} \dot{\xi}_{d}+\zeta \dot{\omega} \geq 0 \text {. }
$$

In order to regulate the activation of each dissipative mechanism, two different yield functions are defined in the space of the proper static variables, namely:

$$
\Phi_{p}\left(\boldsymbol{\sigma}^{c}, \chi_{p}\right) \leq 0, \quad \Phi_{d}\left(\zeta, \chi_{d}\right) \leq 0
$$

where $\Phi_{p}$ is the classical plastic yield function specifying the elastic contact domain assumed convex and $\Phi_{d}$ is the damage activation function also assumed convex.

The activation of each dissipation mechanism can be effectively described by a variational formulation which is represented by the generalized principle of maximum intrinsic dissipation:

$$
\max _{\boldsymbol{\sigma}^{c}, \chi_{p}, \chi_{d}, \zeta}\left(\boldsymbol{\sigma}^{c}: \dot{\boldsymbol{\varepsilon}}^{c p}-\chi_{p} \dot{\xi}_{p}-\chi_{d} \dot{\xi}_{d}+\zeta \dot{\omega}\right)
$$


subject to the constraints (Eq. 23). The Kuhn-Tucker conditions of the maximum constrained problem provide the plastic and damage evolution laws of the interphase:

$$
\begin{aligned}
& \dot{\boldsymbol{\varepsilon}}^{c p}=\dot{\lambda}_{p} \frac{\partial \Phi_{p}}{\partial \boldsymbol{\sigma}^{c}} ; \quad \dot{\omega}=\dot{\lambda}_{d} \frac{\partial \Phi_{d}}{\partial \zeta} \\
& -\dot{\xi}_{p}=\dot{\lambda}_{p} \frac{\partial \Phi_{p}}{\partial \chi_{p}} ; \quad-\dot{\xi}_{d}=\dot{\lambda}_{d} \frac{\partial \Phi_{d}}{\partial \chi_{d}} \\
& \Phi_{p}\left(\boldsymbol{\sigma}^{c}, \chi_{p}\right) \leq 0, \quad \dot{\lambda}_{p} \geq 0, \quad \dot{\lambda}_{p} \Phi_{p}\left(\boldsymbol{\sigma}^{c}, \chi_{p}\right)=0 \\
& \Phi_{d}\left(\zeta, \chi_{d}\right) \leq 0, \quad \dot{\lambda}_{d} \geq 0, \quad \dot{\lambda}_{d} \Phi_{d}\left(\zeta, \chi_{d}\right)=0
\end{aligned}
$$

being $\dot{\lambda}_{p}$ and $\dot{\lambda}_{d}$ the plastic and damage multiplier, respectively.

In the present study the elasto-plastic convex domain is defined by the intersection of the classical Mohr-Coulomb bilinear function with a tension cutoff:

$$
\begin{aligned}
& \Phi_{p 1}\left(\boldsymbol{\sigma}^{c}, \chi_{p}\right)=\left|\boldsymbol{\tau}^{c}\right|+\sigma^{c} \tan \varphi-c_{0}\left(1-\chi_{p}\right) \\
& \Phi_{p 2}\left(\boldsymbol{\sigma}^{c}, \chi_{p}\right)=\sigma^{c}-\sigma_{0}\left(1-\chi_{p}\right)
\end{aligned}
$$

where $\tau^{c}$ and $\sigma^{c}$ are the tangential stress vector and the normal stress component of the contact stresses, $\varphi$ is the friction angle, $c_{0}$ and $\sigma_{0}$ the cohesion and tensile strength of the virgin interfaces.

The two yield functions are depicted in Figure 2. The following four zones can be distinguished:

I elastic zone: $\Phi_{p 1}<0, \Phi_{p 2}<0$

II plastic activation in shear: $\Phi_{p 1}=0, \Phi_{p 2}<0$

III plastic activation in tension: $\Phi_{p 1}<0, \Phi_{p 2}=0$

IV plastic activation in tension and shear (corner): $\Phi_{p 1}=0, \Phi_{p 2}=0$.

The damage activation function is linear and the first activation occurs when the thermodynamic force reaches the relative threshold value $\zeta_{0}$ :

$\Phi_{d}\left(\zeta, \chi_{d}\right)=\zeta-\zeta_{0}\left(1-\chi_{d}\right)$

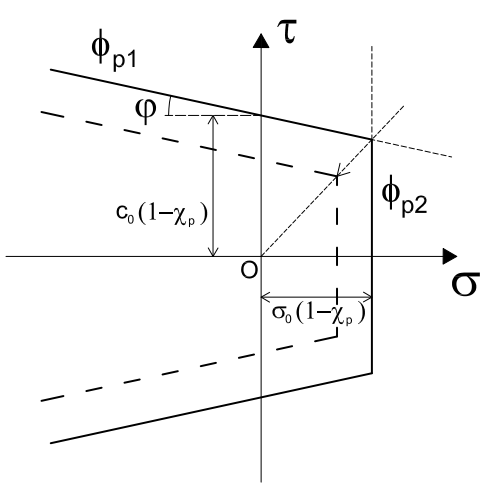

Figure 2. Plastic yield conditions represented in the stress space

\section{NUMERICAL APPLICATIONS.}

The interphase model presented in Sections 2 and 3 has been implemented in an open-source researchoriented finite element analysis program for 2D applications. With the aim to run a step-by-step integration, flow rules have been rewritten as discrete laws. The implicit backward-Euler difference method was applied to obtain results within the time step $\left[t_{n}, t_{n+1}\right] \subset[0, T]$. In particular the nonlinear solution at time $t_{n+1}$ has been calculated by means of an elastic prediction - plastic and/or damaging correction procedure. The interphase element has four nodes and zero-thickness. The integration of the stiffness matrix has been solved by applying the Reduced Selective Integration method, that is two Gauss are used for the integration in the direction normal to the interphase plane while one Gauss point is used in the tangential one.

The numerical applications presented in this work regard diagonal compression test on a cylindrical masonry specimen and a masonry vault loaded by a monotonic increasing load. All numerical examples have been carried out under the hypothesis of plane stress state.

\subsection{Diagonal compression test on masonry.}

A diagonal compression numerical test has been carried out on a masonry panel and compared with the experimental results obtained in laboratory. With reference to Figure 3, the specimen is made of four courses of sandstone blocks with calcium-cement mortar. It has a squared shape with a length of $67 \mathrm{~cm}$ for each side. A single block is $33 \mathrm{~cm}$ long and 16 $\mathrm{cm}$ high. The mortar layer has a thickness of $1 \mathrm{~cm}$. A total number of 256 plane stress 2D solid elements and 72 interphase elements has been used to create the finite element model.

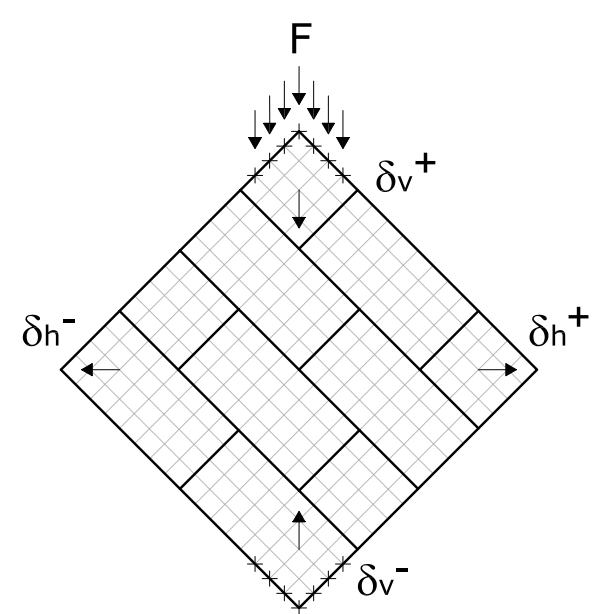

Figure 3. Diagonal compression test on a masonry panel: finite element model. 
Table 1. Parameters used for diagonal compression test.

\begin{tabular}{lll}
\hline Parameter & Brick & Mortar \\
\hline E $(\mathrm{MPa})$ & 11141.67 & 37000 \\
$\mathrm{v}$ & 0.25 & 0.14 \\
$\mathrm{c}_{0}(\mathrm{MPa})$ & - & 5.0 \\
$\sigma_{0}(\mathrm{MPa})$ & - & 1.0 \\
$\zeta_{0}(\mathrm{MPa})$ & - & $10^{-5}$ \\
$\varphi\left({ }^{\circ}\right)$ & - & 35 \\
$\mathrm{~h}_{\mathrm{p}}$ & - & 0.04 \\
$\mathrm{~h}_{\mathrm{d}}$ & - & 0.001 \\
\hline
\end{tabular}

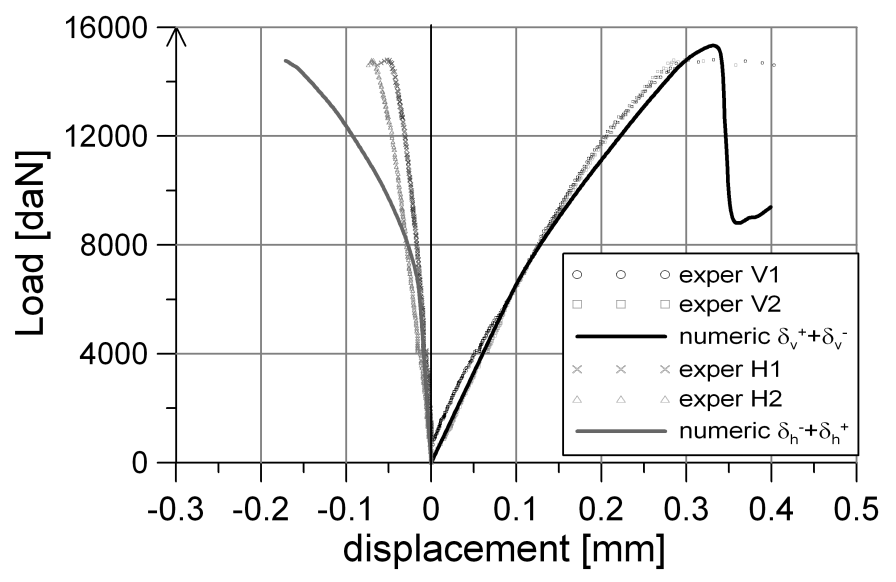

Figure 4. Load-displacement curves: comparison between numerical and experimental results.
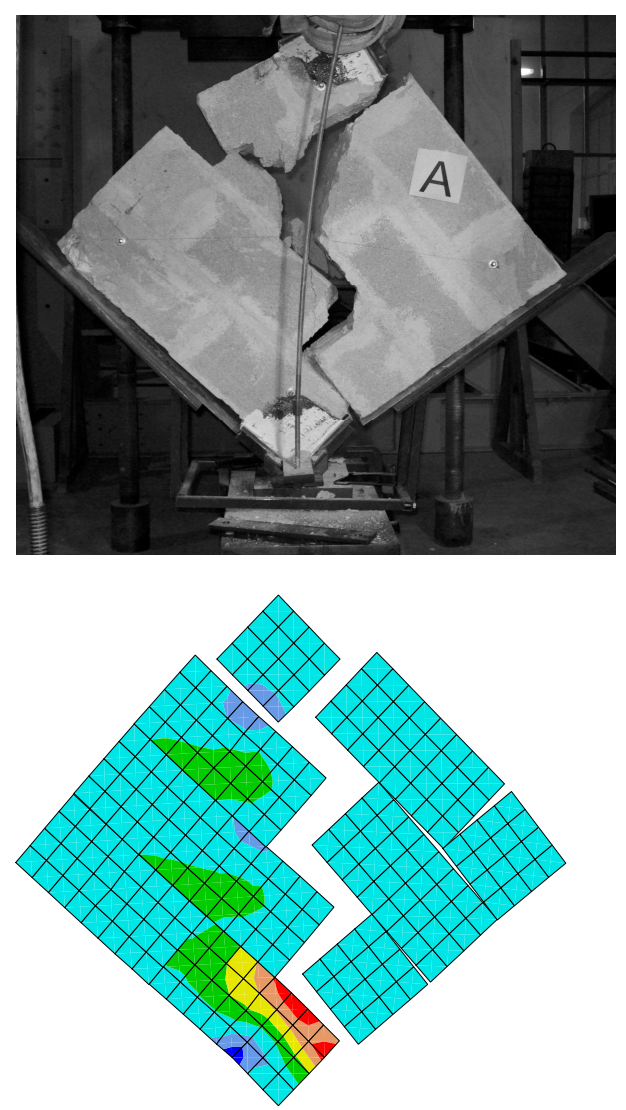

PRIN. STRESS 1

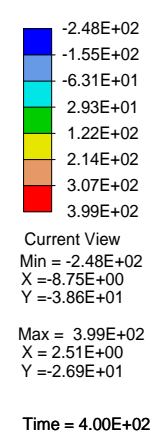

Figure 5. Comparison between numerical and experimental collapse configurations.
The mechanical variables for blocks and mortar and the parameters used for the finite element model are reported in Table 1.

The test has been run with 400 steps under displacement control. At each step the values of the total vertical load $\mathrm{F}$ and the displacement discontinuities $\delta h=\delta h^{+}+\delta h^{-}$and $\delta v=\delta v^{+}+\delta v^{-}, \quad$ in horizontal and vertical direction respectively, have been evaluated and reported in the loaddisplacement curves of Figure 4. A good agreement has been obtained with experimental results.

Figure 5 finally shows a comparison between the collapse experimental and numerical configurations. A very good agreement can be observed, even if the numerical model is not able to simulate the fracture inside the blocks because an elastic behavior was chosen for bricks.

\subsection{Cylindrical masonry vault.}

The second example evaluates the structural response of a cylindrical masonry vault.

The results of the numerical analysis have been compared to the experimental work of Failla et al. (2004) who tested a vault made of 24 courses of calcarenite ashlars stones $\left(21 \times 36 \times 16 \mathrm{~cm}^{3}\right)$. Each course is separated by a mortar layer of average thickness $\mathrm{h}$ equal to $2.5 \mathrm{~cm}$. The vault is $118 \mathrm{~cm}$ long, $16 \mathrm{~cm}$ thick and of internal radius equal to 260 $\mathrm{cm}$. The imposts form an angle of $30^{\circ}$ with the horizontal plane (Fig. 6). The vault has been subjected to a uniform load applied onto the seventh course of stones. The test has been performed under displacement control until the formation of four hinges has been reached.

The structure has been numerically implemented as a bi-dimensional arch, $118 \mathrm{~cm}$ wide. Each stone has been discretized with a 4 x 8 plane stress solid elastic elements (4-noded). The radial joints have been modeled using eight interface elements (4noded).

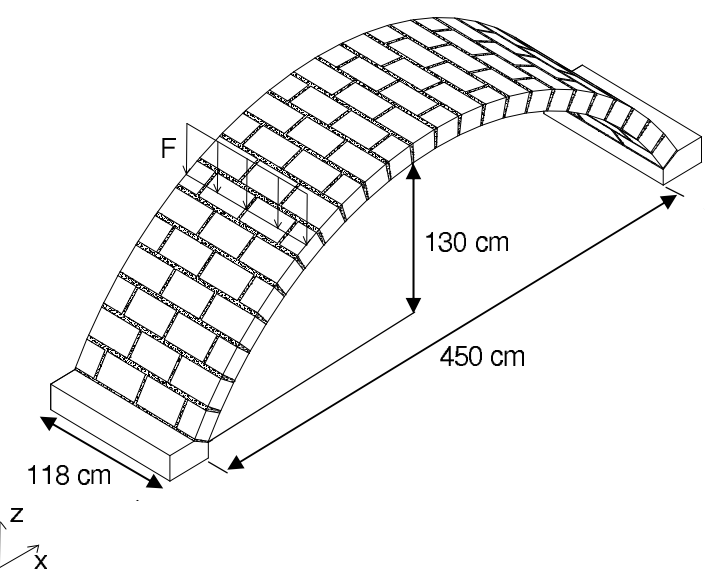

Figure 6. Tridimensional view of the vault. 
To adhere to the experimental protocol, the numerical analysis has been carried out in displacement control.

The load-displacement curve, in comparison with experimental one, is reported in Figure 7. It is clear as the numerical model is able to catch the same stiffness, peak load and residual force of the experimental test. Figure 8 shows instead the final configuration of the vault after the formation of the hinges.

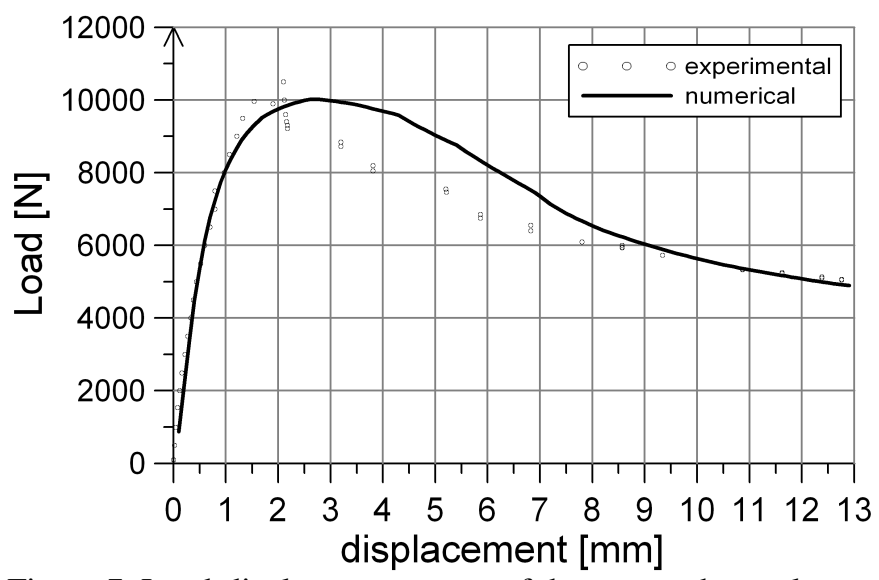

Figure 7. Load-displacement curve of the test on the vault.

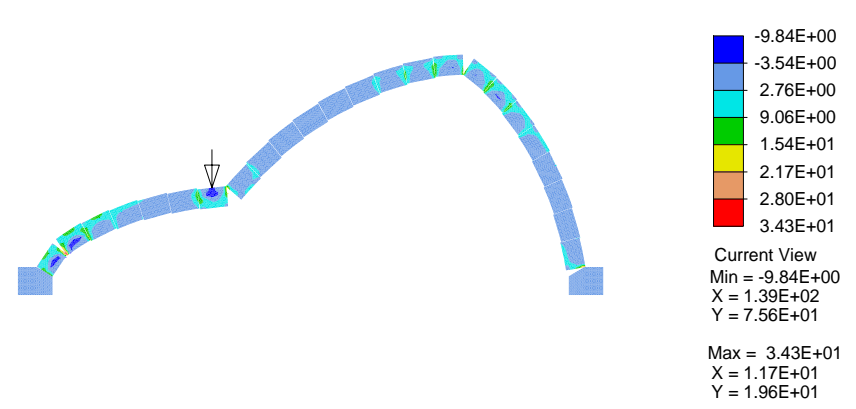

Figure 8. Collapse mechanism of the vault.

\section{CONCLUSIONS.}

The present paper deals with the mesomodelling of heterogeneous structures by means of interphase elements, that can be considered as an enhancement of the common interface elements. Separate failure conditions have been considered for the bulk material and for contact tractions. An isotropic damage model has been used to model the nonlinear response of the bulk material, while an elastoplastic bilinear domain governs the evolution of plasticity for contact tractions. The interphase model has been written in the framework of a thermodynamically consistent theory. State equations and flow rules have been derived and rewritten in a discrete form to be suitable to be used for finite element implementation. Two numerical applications on masonry structural elements have been conducted.
Ongoing and future efforts are devoted to the introduction of plastic activation functions on the physical interfaces between mortar and block, and to the possibility to introduce a new damage model to catch horizontal fractures also.

\section{ACKNOWLEDGEMENTS.}

The authors acknowledge the financial support given by the Italian Ministry of Education, University and Research (MIUR) under the PRIN09 project 2009XWLFKW_005, "A multiscale approach for the analysis of decohesion processes and fracture propagation".

\section{REFERENCES.}

Alfano, G. \& Crisfield, M.A. 2001. Finite element interface models for the delamination analysis of laminated composite: mechanical and computational issues. Int. Journ. Num. Meth. Engrg. 50: 1701-1736.

Alfano, G. \& Sacco, E. 2006. Combining interface damage and fiction in a cohesive-zone model. Int. Journ. Num. Meth. Engrg. 68: 542-582.

Einav, I., Houlsby, G.T. \& Nguyen, G.D. 2007. Coupled damage and plasticity models derived from energy and dissipation potentials. Int. Journ. Solids Struct. 44: 2487-2508.

Failla, A., Cottone, G. \& Giambanco, G. 2004. Numerical modeling of masonry structures reinforced by FRP plate/sheets. In Proceedings of the: Fourth International Seminar on Structural Analysis of Historical Constructions, Padova, 10-13 November 2004. Padova, Italy.

Giambanco, G. \& Mroz, Z. 2001. The interphase model for the analysis of joints in rock masses and masonry structures. Mechanica 36 (1): 111-130.

Giambanco, G., Rizzo, S. \& Spallino, R. 2001. Numerical analysis of masonry structures via interface models. Comput. Methods Appl. Mech. Engrg. 190: 6493-6511.

Giambanco, G., Fileccia Scimemi, G. \& Spada, A. 2012. The interphase element. Comp. Mech. 50 (3): 353-366.

Lofti, H.R. \& Shing, P.B. 1994. Interface model applied to fracture of masonry structures. Journ. Struct. Eng. (ASCE) 120 (1): 63-80.

Lourenco, P.B. \& Rots, J. 1997. Multisurface interface model for analysis of masonry structures. Journ. Engrg. Mech. 123 (7): 660-668.

Parrinello, F., Failla, B. \& Borino, G. 2009. Cohesive-frictional interface costitutive model. Int. Journ. Solids Struct. 46: 2680-2692.

Sacco, E. \& Lebon, F. 2012. A damage-friction interface model derived from micromechanical approach. Int. Journ. Solids Struct. 49 (26): 3666-3680.

Spada, A., Giambanco, G. \& Rizzo, P. 2009. Damage and plasticity at the interfaces in composite materials and structures. Comput. Methods Appl. Mech. Engrg. 198: 3884-3901.

William, K., Rhee, I. \& Shing, B. 2004. Interface damage model for thermomechanical degradation of heterogeneous materials. Comput. Methods Appl. Mech. Engrg. 193: 33273350 . 